\title{
Application of hydroxyapatite nanoparticles in development of an enhanced formulation for delivering sustained release of triamcinolone acetonide
}

\author{
This article was published in the following Dove Press journal: \\ International Journal of Nanomedicine \\ 16 April 2011 \\ Number of times this article has been viewed
}

\section{Saeid Koocheki' \\ Sayed Siavash Madaeni' \\ Parisa Niroomandi ${ }^{2}$ \\ 'Membrane Research Center, Chemical Engineering Department, Razi University, Kermanshah, Iran; ${ }^{2}$ Exir Pharmaceutical Co, Lorestan, Boroujerd, Iran}

Correspondence: Sayed Siavash Madaeni Membrane Research Center, Chemical Engineering Department, Razi University, Baghe Abrishan, 67I44 Kermanshah, Iran Tel $+9883 \mid 4274530$

Fax +98 83I 4274542

Email smadaeni@yahoo.com

\begin{abstract}
We report an analysis of in vitro and in vivo drug release from an in situ formulation consisting of triamcinolone acetonide (TR) and poly(D,L-lactide-co-glycolide) (PLGA) and the additives glycofurol (GL) and hydroxyapatite nanoparticles (HA). We found that these additives enhanced drug release rate. We used the Taguchi method to predict optimum formulation variables to minimize the initial burst. This method decreased the burst rate from $8 \%$ to $1.3 \%$. PLGA-HA acted as a strong buffer, thereby preventing tissue inflammation at the injection site caused by the acidic degradation products of PLGA. Characterization of the optimized formulation by a variety of techniques, including scanning electron microscopy, X-ray diffraction, differential scanning calorimetry, and Fourier transform near infrared spectroscopy, revealed that the crystalline structure of TR was converted to an amorphous form. Therefore, this hydrophobic agent can serve as an additive to modify drug release rates. Data generated by in vitro and in vivo experiments were in good agreement.
\end{abstract}

Keywords: triamcinolone acetonide, glycofurol, hydroxyapatite nanoparticle, PLGA

\section{Introduction}

Injectable drug delivery systems have improved significantly. ${ }^{1-3}$ These improvements make them easier to use, enable targeted delivery, ${ }^{4-6}$ optimize delivery periods, minimize side effects by reducing drug dose, and increase patient comfort and compliance. ${ }^{7}$ Creating in situ implants has become a frequently used technique, by which the drug and a biodegradable polymer are dissolved in an organic solvent and administered by subcutaneous injection, generating a semi-solid depot. This drug delivery system is a good alternative to other implant devices for the following reasons: the process causes much less pain in comparison to implants, which require local surgery; localized or systemic drug delivery can be maintained for months; 'infusion-like' plasma level time profiles minimize side effects. Other advantages include dose reduction to avoid peaks and valleys of drug concentration in blood, and enhanced patient compliance by reducing application frequency. In situ forming depot systems using polymers are relatively simple to produce and cost less than microspheres, which have to be washed and isolated. ${ }^{8}$

There are two different techniques for delivering in situ forming implants and in situ forming microparticles, depending on depot type. An in situ forming implant consists of a drug-containing biodegradable polymer solution in a biocompatible organic solvent. The solvent diffuses in the body's aqueous environment, leading to 
polymer precipitation and formation of an implant at the injection site. The most popular and biocompatible solvent used is N-methyl-2-pyrrolidone (NMP). ${ }^{9-13}$ Poly(D,L-lactide-co-glycolide) (PLGA) is the most successfully used biodegradable and biocompatible polymer because of its two biodegradable hydrolysis products, lactic and glycolic acids (Figure 1). ${ }^{14}$

The properties of PLGA that affect drug release, including its molecular weight (MW), lactide/glycolide ratio, and thermal properties, have been thoroughly investigated. Using lower MW PLGA increases the rates of polymer degradation and drug release. Decreasing the lactide content results in slower polymer degradation and slower drug release. ${ }^{15}$

Experimental design involves defining and investigating all factors thought to affect the outcome, ${ }^{16}$ and experiments are executed together or sequentially to optimize the variable parameters and define the optimum conditions. In situations involving numerous variables, a method called factorial design is used to discover the best combination of control factors to increase efficiency and reduce costs. ${ }^{17}$ However, this may not be possible in cases of high complexity. ${ }^{18}$ In the present study, we evaluated the parameters affecting the release rate of triamcinolone acetonide (TR) loaded on PLGA in the presence of other additives. Process parameters were optimized using the robust Taguchi method, which provides a comprehensive system for improving and optimizing drug release by considering equal contributions of variables. ${ }^{19}$ The resulting optimized formulation was characterized using scanning electron microscopy (SEM), X-ray diffraction (XRD), differential scanning calorimetry (DSC), and Fourier transform near infrared spectroscopy (FTNIR).

TR is a steroidal drug usually administered by the parenteral route (Figure 2). Its release from a biodegradable PLGA polymer can be evaluated by the two key parameters of burst release rate and release duration. These parameters are affected by drug loading, which is considered the main focus for in vivo burst release optimization.

\section{Materials and methods}

\section{Chemicals}

The following chemicals were used as received from the suppliers: PLGA, Resomer $^{\circledR}$ RG 504H (MW = 48,000 kDa)

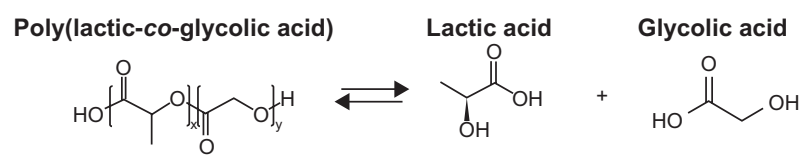

Figure I Poly(D,L-lactide-co-glycolide) hydrolysis.

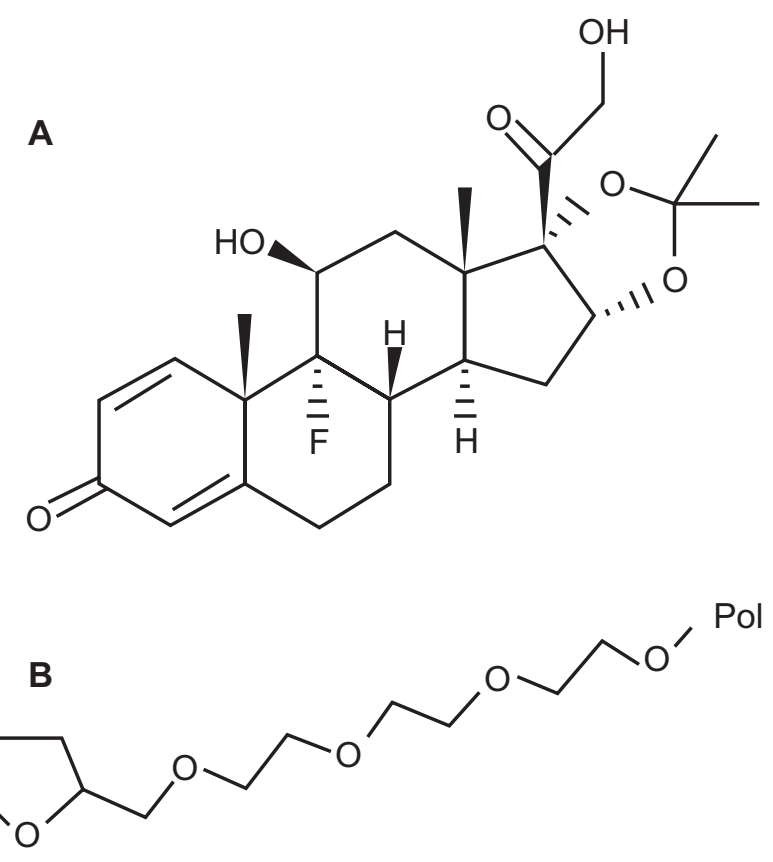

Figure 2 Structures of A) triamcinolone acetonide and B) polyethylene glycol ether.

(Boehringer Ingelheim, Ingelheim, Germany), TR (Farmabios, Gropello Cairopoli, Italy), NMP (Merck KGaA, Darmstadt, Germany), methanol (HPLC Gradient Grade, Merck KGaA), acetonitrile (HPLC Gradient Grade, Merck KGaA), $\mathrm{NaOH}$ (Merck KGaA), tetrahydrofurfuryl alcohol, polyethylene glycol ether (GL) (glycofurol, Merck KGaA), and hydroxyapatite (HA) nanoparticles (Sigma-Aldrich, Munich, Germany).

\section{Methods and characterization}

\section{Casting solution preparation}

Homogeneous casting solutions $(33 \% \mathrm{w} / \mathrm{w})$ were prepared from PLGA in NMP. Varying amounts of drug were added and completely dissolved before introducing additives (Table 1). To study drug release patterns, experiments were carried out in glass vials type I $(15 \mathrm{~mL})$ (Schott Duran ${ }^{\circledR}$, Mainz, Germany), because they possess the lowest available drug absorption properties. Vials were charged with the casting solutions, weighed immediately, and then filled with

Table I Selected parameters and their levels for experimental design

\begin{tabular}{llll}
\hline Parameters & \multicolumn{2}{l}{ Level } \\
\cline { 2 - 4 } & I & $\mathbf{2}$ & $\mathbf{3}$ \\
\hline A) TR\% (w/w) & 5 & 10 & 15 \\
B) Nano HA\% (w/w) & I & 3 & 5 \\
C) GL\% (w/w) & I & 3 & 5 \\
\hline Abbreviations: TR trian
\end{tabular}

Abbreviations: TR, triamcinolone acetonide; $\mathrm{HA}$, hydroxyapatite; $\mathrm{GL}$, polyethylene glycol ether. 
$10 \mathrm{~mL}$ degassed phosphate buffer (PB) $(0.03 \mathrm{M}, \mathrm{pH}=7.4)$ to minimize solvent exchange with air. All vials were incubated at $37 \pm 0.5^{\circ} \mathrm{C}$. The entire receptor phase $(10 \mathrm{~mL})$ was withdrawn at predetermined intervals and replaced by the same amount of freshly prepared receptor medium.

\section{Release measurements}

Formulation performance was evaluated by measuring TR release kinetics using a reverse phase high performance liquid chromatography (HPLC) method (C-18 column, 4.6×250 mm; NovaPak $^{\circledR}$; Waters, Milford, MA). The isocratic mobile phase consisted of a mixture of deionized water, acetonitrile, and methanol (62:33:5). Ultraviolet light absorbance was measured at $254 \mathrm{~nm}$, and the column temperature was maintained at $25^{\circ} \mathrm{C}$. Each experiment was performed in triplicate.

\section{Formulation optimization}

The experiments were designed using the Taguchi method. In order to determine the best formulations, three parameters, including TR, HA nanoparticles, and GL, as well as three levels for each parameter (Table 1) and a fractional factorial design, namely, a standard $\mathrm{L}_{9}$ orthogonal array, ${ }^{20}$ were employed (Table 2). This orthogonal array was selected to reduce interaction among the parameters. Each row of the matrix represents one run. The sequence of these runs is randomized. As shown in Table 1, three different levels related to each parameter are represented as ' 1 ', ' 2 ', and ' 3 '. QUALITEK-4 software (Nutek, Bloomfield Hills, MI) was used for experimental design and data analysis. ${ }^{21}$ Results are expressed as the average of the three analyses.

\section{SEM}

Formulation surfaces were studied with a scanning electron microscope, VEGA 3 SB (TESCAN, as, Brno, Czech Republic). Gold-plated samples were examined at room temperature.

Table $2 \mathrm{~L}_{9}$ orthogonal array

\begin{tabular}{llll}
\hline Run \# & \multicolumn{2}{c}{ Parameter levels } & C \\
\cline { 2 - 4 } & A & B & I \\
\hline I & I & I & 2 \\
2 & 1 & 2 & 3 \\
3 & 1 & 3 & 2 \\
4 & 2 & 1 & 3 \\
5 & 2 & 2 & 1 \\
6 & 2 & 3 & 3 \\
7 & 3 & 1 & I \\
8 & 3 & 2 & 2 \\
9 & 3 & 3 & \\
\hline
\end{tabular}

XRD

The XRD patterns of the formulations were measured using a PANalytical X-ray diffractometer (XPERT PRO, PW 3040/60 generator, PIXcel Detector and X'Pert Data Collector software for automatic powder diffraction, v 2.2i; PANalytical, Almelo, the Netherlands). The X-ray diffractometer was operated with an anode current of $40 \mathrm{~mA}$ and an accelerating voltage of $40 \mathrm{kV}$. Samples were slightly pressed on an aluminium sample tray using a glass slide $(75 \times 25 \mathrm{~mm})$ and exposed to $\mathrm{CuK} \alpha$ radiation at diffraction angles $(2 \theta)$ from $5^{\circ}$ to $40^{\circ}$ (step size, $0.02^{\circ}$; time per step, 40.01 seconds). X'Pert HighScore Plus (v 2.2d; PANalytical) software was used to locate the peaks in XRD diffractograms by detecting second derivative minima. Intensity maxima are expressed as $\mathrm{K} \alpha 1$ net peak height in counts at $\mathrm{K} \alpha 1$ position in degrees.

\section{DSC}

A Perkin-Elmer DSC 7 (PerkinElmer, Skovlunde, Denmark) with Pyris software was used to determine the glass transition temperature (Tg). The sample (1.5-3.0 mg) was transferred to an aluminium pan in which a pinhole was punched in the pan lid, and the sides of the lid were crimped. A similar empty pan was used as reference. Samples were scanned from $10-70^{\circ} \mathrm{C}$ at a rate of $10^{\circ} \mathrm{C} /$ minute under nitrogen gas (20 $\mathrm{mL} /$ minute). Independent triplicate measurements are expressed as their means. The instrument was calibrated using an indium standard.

\section{FTNIR}

Infrared spectra were determined using a Bruker IRspectrometer Tensor 37 (Bruker, Ettlingen, Germany) in single beam transmittance mode with optical probe. Each spectrum consisted of 32 scans collected in single beam at a resolution of $4 \mathrm{~cm}^{-1}$ at $25^{\circ} \mathrm{C}$. Spectra were obtained on a straight baseline in the near infrared (NIR) region.

\section{Hot plate method}

The hot plate test was performed in mice to assess the release response of triamcinolone. The hot plate test was performed by placing mice on an aluminum plate maintained at $51 \pm 1^{\circ} \mathrm{C}$. The latency to an analgesic response, identified as licking of either hind paw, or lifting of either hind paw from the heated surface, was measured.

\section{Results and discussion Data analysis}

Figure 3 shows the release profiles of TR from different formulations of PLGA-based implants. In the present study, 


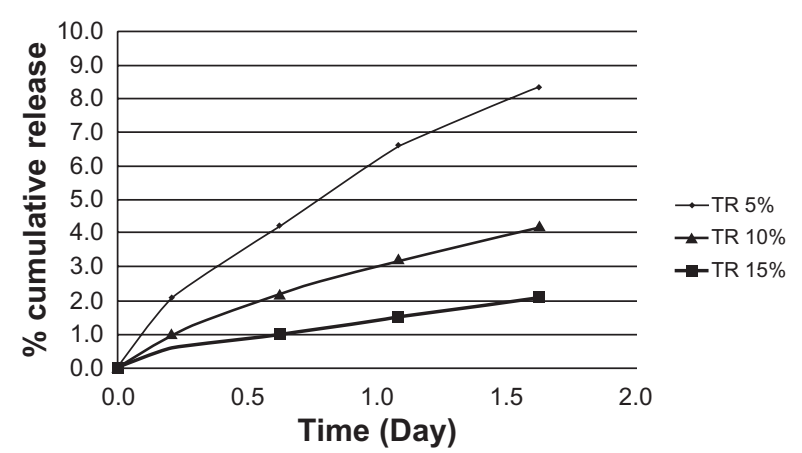

Figure 3 Triamcinolone acetonide release kinetics in poly(D,L-lactide-co-glycolide) in phosphate buffer.

to reduce the burst release associated with PLGA-based delivery systems formed in situ, we prepared nine different formulations incorporating TR and the additives described in Table 1. Experiments were executed based on the Taguchi design (Table 2).

Table 3 presents the release rates of a variety of formulations containing different levels of additives. All additives, especially HA, improved release rates. HA naturally occurs in mineral form known as calcium apatite, whose formula, $\mathrm{Ca}_{5}\left(\mathrm{PO}_{4}\right)_{3}(\mathrm{OH})$, is usually written as $\mathrm{Ca}_{10}\left(\mathrm{PO}_{4}\right)_{6}(\mathrm{OH})_{2}$ to denote that the crystal unit cell includes 2 entities. HA is the hydroxyl-end member of the complex apatite group. The SEM image of HA (Figure 4) revealed that these non-sintered nanoparticles are nanocrystal clusters with equally spherical shape. ${ }^{22}$ Most of the drug-loaded formulations showed a biphasic release pattern (Figure 5) exhibiting an initial burst followed by sustained release..$^{23,24}$ The high initial release may be due to the presence of free and weakly bound drug on the surface of carriers.

\section{Prediction, optimization and characterization}

To identify the main parameters' influence on burst release, calculations were based on release data over 1.5 days

Table 3 Cumulative release after 1.5 days

\begin{tabular}{llllll}
\hline Run \# & \multicolumn{2}{l}{ \% response } & & Average (\%) & SD \\
\cline { 2 - 4 } & $\mathbf{I}$ & $\mathbf{2}$ & $\mathbf{3}$ & & \\
\hline 1 & 4.5 & 4.0 & 4.1 & 4.2 & 0.3 \\
2 & 3.9 & 4.1 & 4.7 & 4.2 & 0.4 \\
3 & 4.7 & 4.6 & 4.2 & 4.5 & 0.3 \\
4 & 2.0 & 2.3 & 2.0 & 2.1 & 0.2 \\
5 & 2.2 & 2.3 & 2.2 & 2.2 & 0.1 \\
6 & 2.3 & 2.5 & 2.4 & 2.4 & 0.1 \\
7 & 1.5 & 1.6 & 1.6 & 1.5 & 0.1 \\
8 & 1.4 & 1.5 & 1.3 & 1.4 & 0.1 \\
9 & 1.2 & 1.5 & 1.3 & 1.3 & 0.2 \\
\hline
\end{tabular}

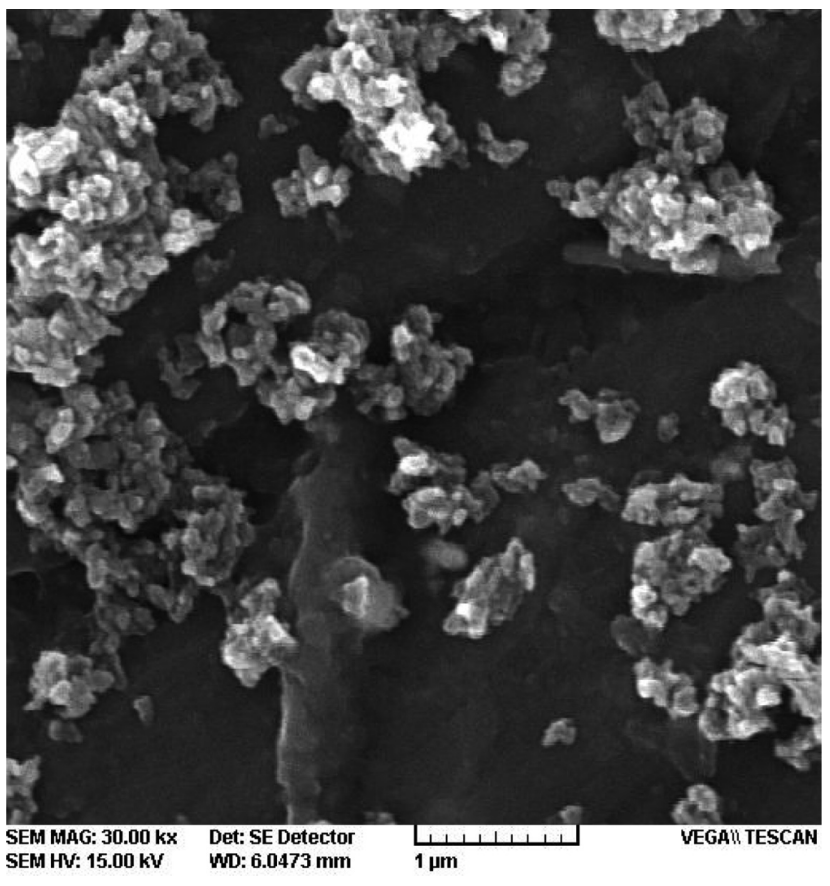

Figure 4 Hydroxyapatite nanoparticles.

(Figure 6). We used QUALITEK-4 software for this analysis (Table 3). In the Taguchi method, the main effect of control parameters indicates the trend of a parameter's influence. The key effects were calculated using average release. The results indicate the drug loading effect on the release profile (Figure 7). Another technique for optimization of the results suggested by the Taguchi method is analysis of variance (ANOVA). Table 4 presents the analysis of variance (ANOVA) values, which indicate the relative influence of factor and interaction to the variation of results. ANOVA is similar to regression analysis, which is used to investigate and determine the relationship between a response variable and one or more independent variables. ${ }^{21}$ Furthermore, Bese et $\mathrm{a}^{25}$ state that optimal combination of process parameters can be predicted from the performance characteristics and

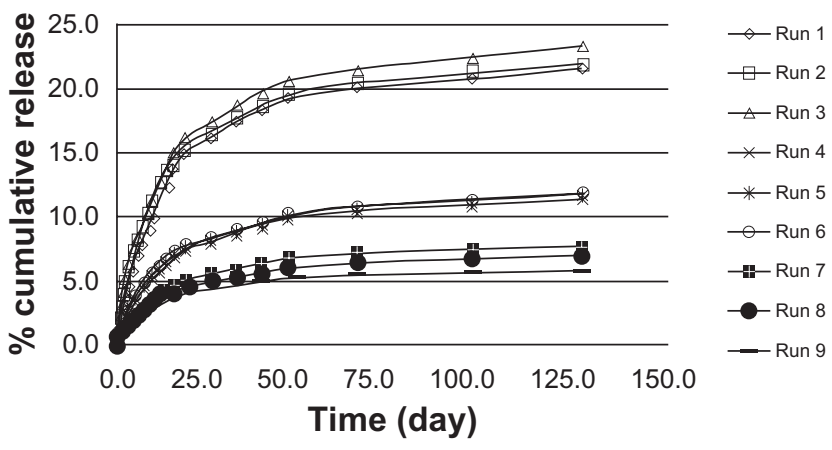

Figure 5 Cumulative triamcinolone acetonide release kinetics in poly(D,L-lactideco-glycolide)-hydroxyapatite in phosphate buffer. 


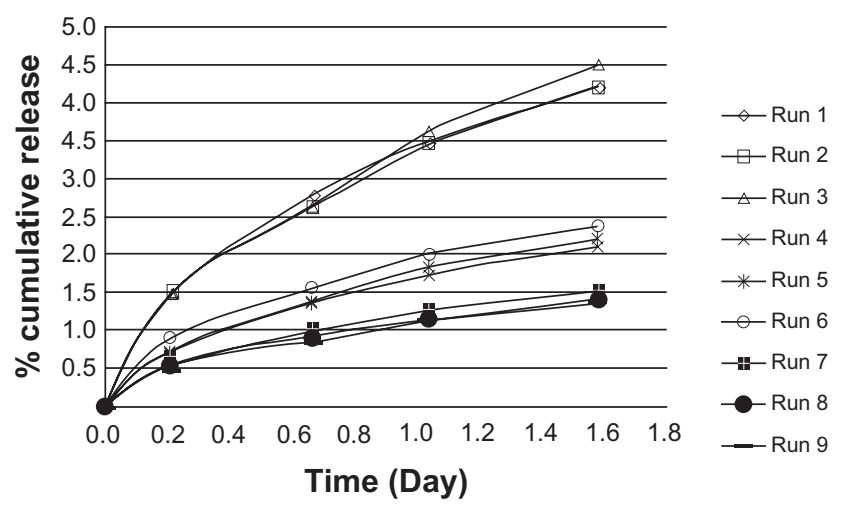

Figure 6 Cumulative TR release kinetics in poly(D,L-lactide-co-glycolide)hydroxyapatite in phosphate buffer (after I.5 days).

ANOVA analyses. The contributions of each parameter to burst release optimization and expected optimum condition are listed in Table 5. According to the Taguchi method, TR had the highest contribution to the decrease of burst release. The highest percentage for control parameters was obtained with the following composition: TR (15\%), HA (1\%), and GL (3\%).

At optimal conditions, burst release decreased to $1.3 \%$. The drug contents in PLGA and PLGA-HA based formulations were $5 \%, 10 \%$, and $15 \%$ (drug/PLGA). These starting values of TR for PLGA and PLGA-HA formulations were taken as the $100 \%$ starting values of the released drug. TR release from PLGA (Figure 3) and PLGA-HA (Figure 6) was conducted in $\mathrm{PB}$ ( $\mathrm{pH}$ 7.4). As can be seen, the release profiles of the two formulations are different (Figures 3 and 6). The PLGA release profile is characterized by a biphasic drug release typically exhibited by biodegradable polymers: an initial burst followed by a sustained release. However, PLGA-HA displayed a triphasic drug release. During the first 1.5 days, a small 'burst' was observed for both PLGA (8\%) and PLGA-HA (4.5\%) based on the total amount of loaded drug. The initial burst was caused by the drug's rapid release

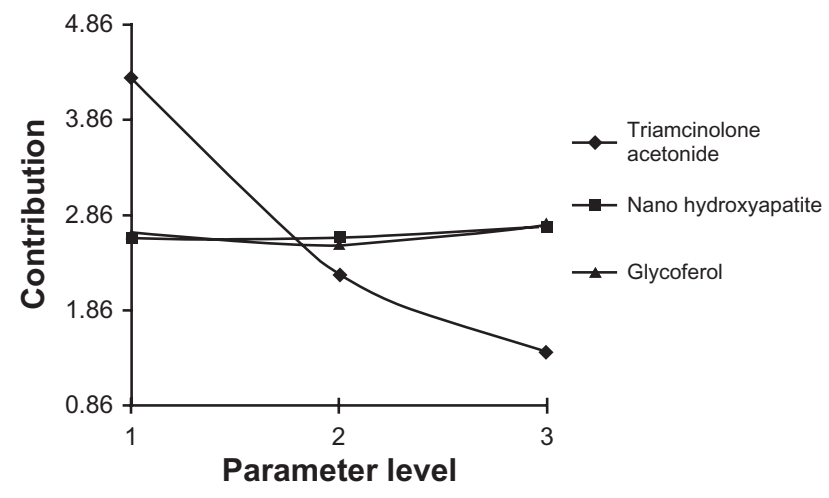

Figure 7 Effects of variables vs parameter levels. near the surface. The second plateau phase for PLGA-HA lasted around 20 days with a low dose of TR released. The plateau phase was followed by a third stage with decreasing drug release lasting for over 100 days. A gradually increased release during the first 20 days was followed by a slow release at a nearly constant rate of $0.05 \%$ per day for PLGA-HA. The cumulative drug release profile of TR (15\%) from PLGA was similar to that of PLGA-HA. These release rates were 1.5 and 2 times that of $10 \%$ and $5 \%$ TR, respectively (Figures 3 and 6). These results show that at 15\% TR, its release is independent of additive. In contrast, HA controlled the release rate of $5 \%$ and $10 \% \mathrm{TR}$.

Triphasic drug release follows the following stages: initial burst, followed by drug diffusion, with polymer degradation occurring at the third phase. The second phase, which continues for about 20 days, is governed by swelling of the PLGA in situ forming implant. TR is released slowly from the in situ gel, and is replaced by water. The precipitation of PLGA follows a biphasic mechanism. After injection, a thin PLGA membrane is immediately formed at the injection site. Dissolved NMP and TR diffused through this polymer shell into the aqueous environment, and water diffused into the polymer, simultaneously. As hardening of the PLGA membrane increases due to further polymer precipitation, the diffusion distances are increased. According to the EinsteinSmoluchowski equation (1), where D, d, and t are the diffusion coefficient, distance and time, respectively, as diffusion distances inside the implant are increased, the diffusion times for NMP outside, and water inside, are increased. ${ }^{26,27}$ This is represented by the following equation:

$$
D=\frac{d^{2}}{2 t} \rightarrow t=\frac{d^{2}}{2 D}
$$

During the formation of implants of PLGA and PLGA-HA in situ in $\mathrm{PB}$, the $\mathrm{pH}$ decreased to approximately 5 and 6 , respectively. In the presence of $\mathrm{HA}$, the $\mathrm{pH}$ decreased more slowly (Figure 8). Lactic and glycolic acids are two products that are generated by PLGA biodegradation and cause tissue inflammation at the injection site. Compared to PLGA, PLGA-HA demonstrated significant buffer capacity. This anti-inflammatory property, which was expected from this formulation, is in accordance with other studies. ${ }^{28,29}$ After 20 days, while the PLGA formulation was extensively degraded (Figure 9A), PLGA-HA was only partially disrupted (Figure 9B). Slower degradation of PLGA-HA is due to the presence of HA. Meanwhile, this additive caused a low burst and controlled the overall rate of drug release as shown in 
Table 4 ANOVA for cumulative release after 1.5 days

\begin{tabular}{lllllll}
\hline Parameters & DOF & Sum of squares & Variance & F-ratio & Pure sum & Percent (\%) \\
\hline A) TR\% (w/w) & 2 & 39.6 & 19.8 & 450.0 & 39.5 & 96.9 \\
B) Nano HA\% (w/w) & 2 & 0.1 & 0.0 & 1.0 & 0.0 & 0.0 \\
C) GL\% (w/w) & 2 & 0.2 & 0.1 & 2.3 & 0.1 & 0.3 \\
Other/error & 20 & 0.9 & 0.0 & & & 2.8 \\
Total & 26 & 40.8 & & & 100.0 \\
\hline
\end{tabular}

Abbreviations: TR, triamcinolone acetonide; HA, hydroxyapatite nanoparticles; GL, polyethylene glycol ether.

Figure 5. HA as a bisphosphonated and chelating component can be encapsulated into the PLGA matrix. This non-mutually exclusive hypothesis can be proposed as a reason for the slow degradation of PLGA-HA. In addition, HA degradation in $\mathrm{pH}$ $<4.2$ has been investigated previously. ${ }^{29}$ This biodegradation releases $\mathrm{Ca}^{2+}$ and the result is $\mathrm{pH}$ buffering capacity. This can be combined with the anti-inflammatory properties of TR for better control of inflammation due to PLGA hydrolysis at the injection site, using this optimized formulation.

Glycofurol is a water-miscible solvent which is used in the preparation of parenteral pharmaceutical dosage forms and with PLGA for in situ gel forming. ${ }^{7,30}$ This nontoxic solvent enhances the solubility and permeability of water-insoluble drugs. ${ }^{31,32}$ XRD data evaluation leads to the conclusion that TR forms a crystalline structure, which becomes somewhat degraded in the PLGA matrix. This structure was converted to amorphous TR in the presence of 3\% GL. This transition results from hydrogen bonding between TR and GL (Figure 10). The solubility of amorphous TR in intrinsically amorphous PLGA is greater than that of crystalline TR. This increases the density of the membrane structure (Figure 11A) and leads to lower burst release. In the absence of GL, upon phase inversion, TR remains crystalline, and some of these crystals escape from the membrane (Figure 11B) causing a burst release. GL increases the solubility of TR, increasing its bioavailability, consistent with studies by Bakarat. ${ }^{33}$

The structure of polymeric materials can be identified by combinations and overtone vibration of $\mathrm{X}-\mathrm{H}$ bonds using

Table 5 Optimum conditions for the burst release reduction

\begin{tabular}{llll}
\hline Parameter & Level description & Level & Contribution \\
\hline A) TR\% (w/w) & 15 & 3 & 1.230 \\
B) Nano HA\% (w/w) & 1 & 1 & $0.04 \mathrm{I}$ \\
C) GL\% (w/w) & 3 & 2 & 0.108
\end{tabular}

Total contribution from all parameters: 1.380

Current grand average of performance: 2.662

Expected result at optimum condition: 1.283

Abbreviations: TR, triamcinolone acetonide; HA, hydroxyapatite nanoparticles; $\mathrm{GL}$, polyethylene glycol ether. near infrared (NIR) absorption. Hydrogen bonds in $\mathrm{O}-\mathrm{H}$ groups give rise to sharp peaks in infrared (IR) spectroscopy. ${ }^{34}$ The Fourier Transform infrared spectroscopy (FTIR) spectrum revealed interaction between PLGA and amorphous TR, which decreases burst release (Figure 12).

DSC analysis of TR shows endothermic peaks in the $242-280^{\circ} \mathrm{C}$ range, which is characteristic of polymorph $\mathrm{A}$ and $\mathrm{B} .{ }^{35,36}$ The glass transition temperature $\left(\mathrm{T}_{\mathrm{g}}\right.$ ) of PLGA in situ forming gels, including TR (15\%) and GL (3\%), was $49.8^{\circ} \mathrm{C}$. This is also evidence for the transition of TR from a crystalline to an amorphous form in the presence of GL, and the existence of elastic properties in the PLGA membrane. ${ }^{31}$ Solubilization by GL causes this, decreases the burst release, and makes a dense structure of the PLGA implant.

\section{In vivo studies}

Drug release through PLGA has been studied in detail. ${ }^{37-41}$ The purpose of the present study is to systematically incorporate specific excipients into TR-loaded PLGA in situ gel formulations in order to evaluate the burst effect in vivo. The hot-plate test was administered to mice to assess their response to TR release. The latency of an analgesic response was identified as either licking or lifting of a hind paw from the heated surface. A preliminary study of the time course of TR's analgesic activity in mice was performed to determine the time to peak analgesic activity and effective dose $50\left(\mathrm{ED}_{50}\right)$. Eight groups of mice (five mice per group),

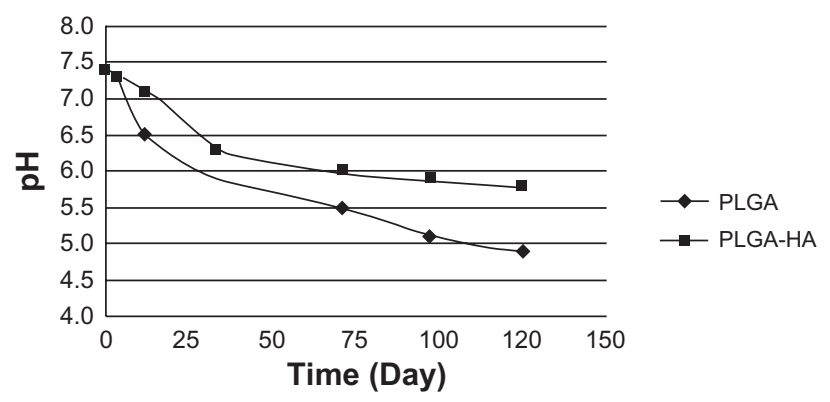

Figure 8 Effects on $\mathrm{pH}$ of triamcinolone acetonide (5\%) with PLGA or PLGA-HA. Abbreviations: PGLA, poly(D,L-lactide-co-glycolide); PGLA-HA, poly(D,L-lactideco-glycolide) with hydroxyapatite. 


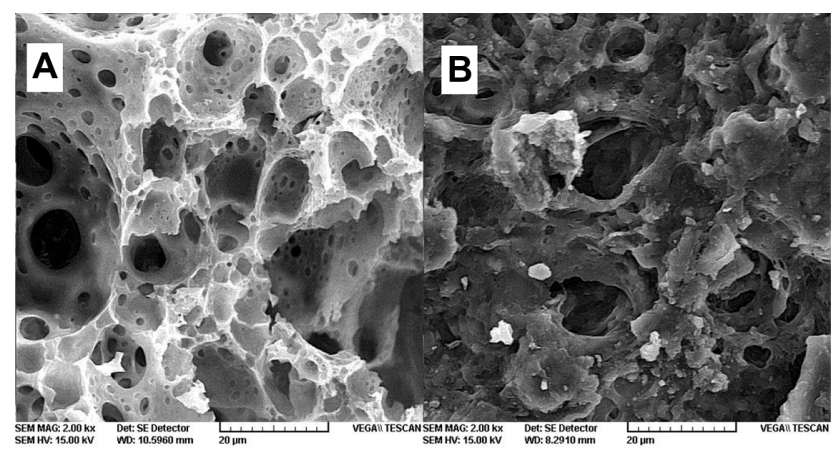

Figure 9 SEM image of membranes after immersion in phosphate buffer for 20 days: A) poly(D,L-lactide-co-glycolide) and B) PGLA-HA: poly(D,L-lactide-co-glycolide) with hydroxyapatite.

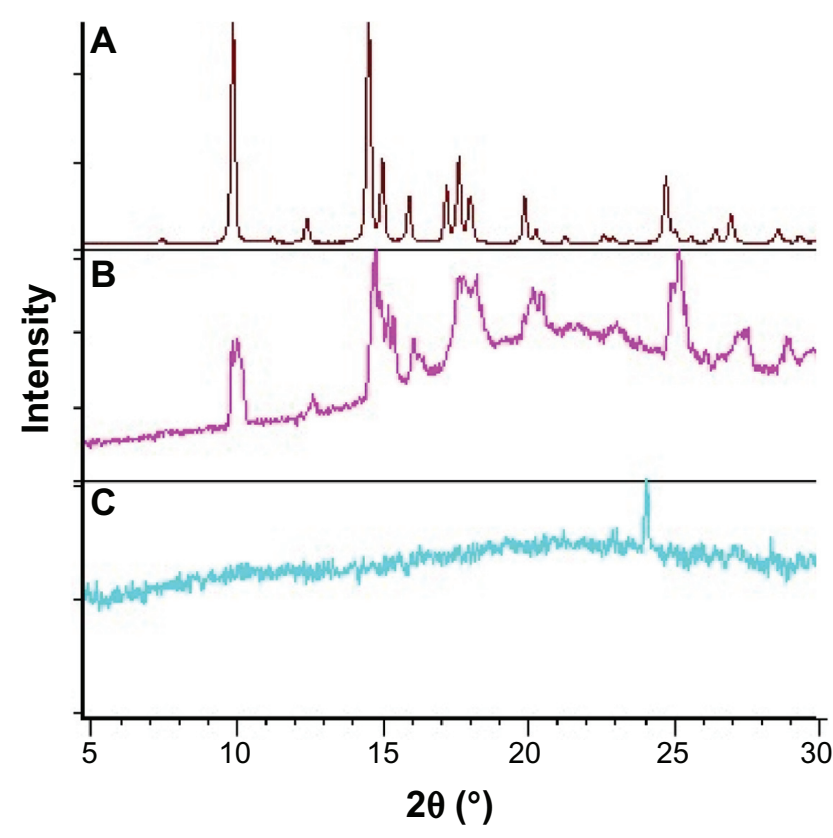

Figure 10 Diffractograms of A) triamcinolone acetonide B) PLGA and triamcinolone acetonide (15\%), C) PLGA and triamcinolone acetonide (15\%) and polyethylene glycol ether (3\%).

Abbreviation: PLGA, poly(D,L-lactide-co-glycolide).

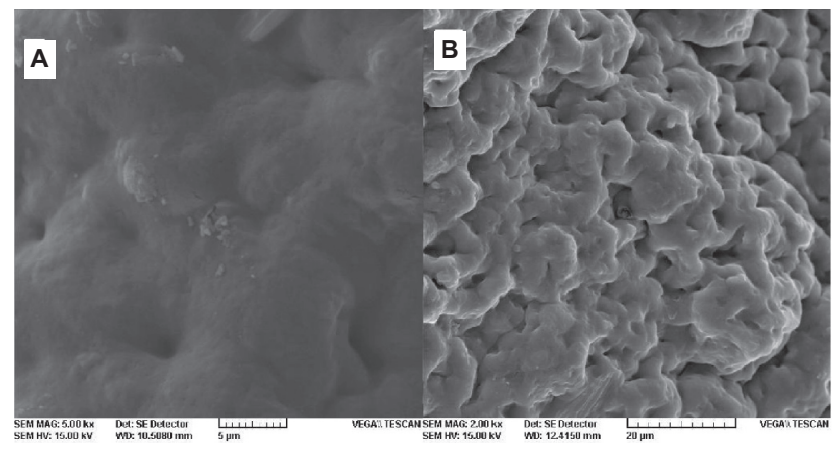

Figure I I Scanning electron microgram of poly(D,L-lactide-co-glycolide) membranes: A) $3 \%$ and B) $0 \%$ polyethylene glycol ether.

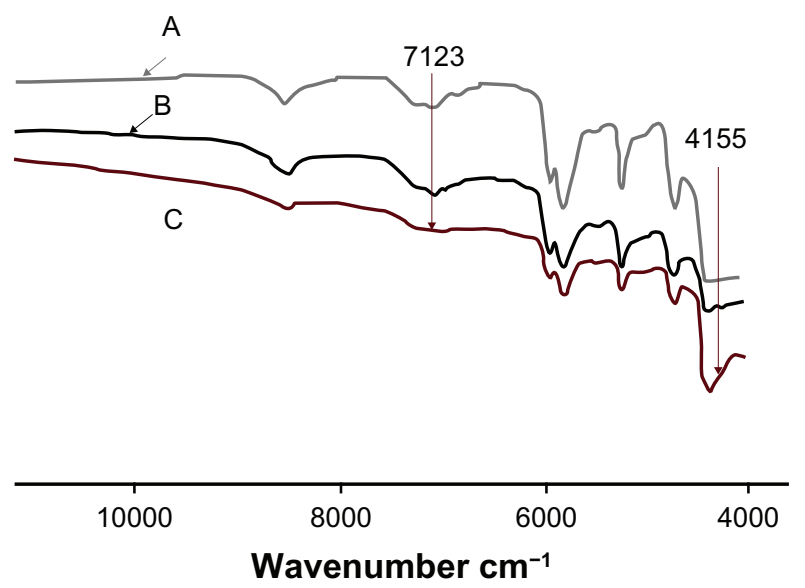

Figure 12 Fourier transform near infrared spectra of poly(D,L-lactide-co-glycolide) membranes in the presence of A) $0 \%$, B) $1 \%$, and C) $3 \%$ polyethylene glycol ether.

designated as group A and B, were selected. TR $(0.01 \mathrm{mg} / \mathrm{kg}$, $0.05 \mathrm{mg} / \mathrm{kg}, 0.1 \mathrm{mg} / \mathrm{kg}$ and $0.5 \mathrm{mg} / \mathrm{kg}$ ) with and without PLGA was injected intramuscularly into members of group $\mathrm{A}$ and $\mathrm{B}$, respectively. Latency time was measured at 30 , 60, 90, 120, 150, and 180 minutes after administration. Injections without PLGA caused a significant increase in reaction times (Figure 13), while PLGA injections caused only a small change (Figure 14). The increase in reaction time was dose-dependent and differed significantly among the groups of mice receiving different TR doses. The data demonstrate that the burst release of TR from the PLGA matrix was very low and that a sustained release of the drug was achieved. Overall evaluation of these results shows that the data are quite comparable to those acquired in vitro. The burst release was decreased and the TR concentration had the greatest effect on control of the burst effect. Thus, we conclude that PLGA injected into the body acts as a depot for the sustained release of TR.

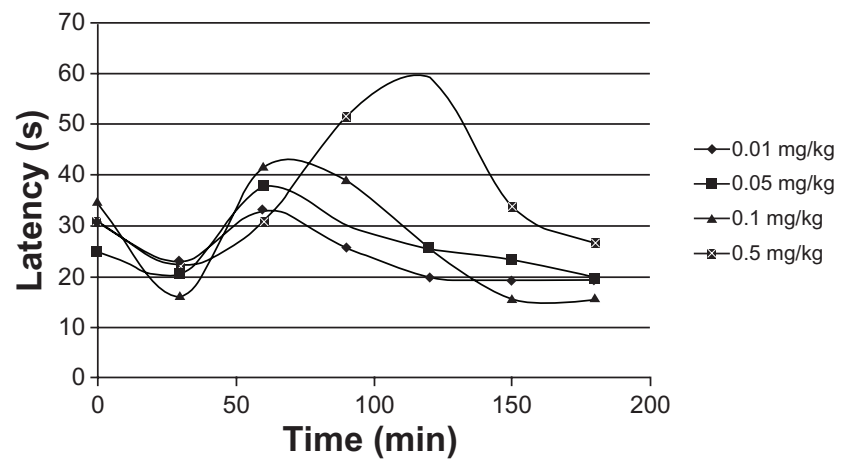

Figure 13 Hot-plate response of mice $(n=5)$ administered triamcinolone acetonide. 


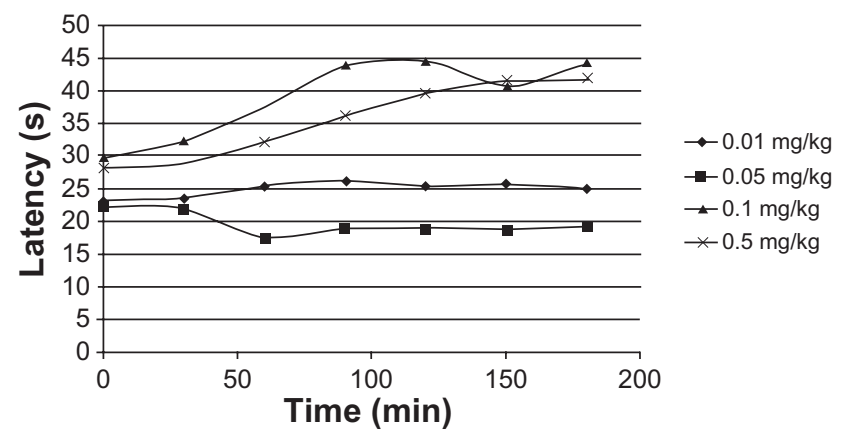

Figure 14 Hot-plate response of mice $(n=5)$ administered triamcinolone acetonide with poly(D,L-lactide-co-glycolide).

\section{Conclusion}

We applied the Taguchi method to predict and optimize in situ gel formulation of TR and PLGA polymer in the presence of HA nanoparticles and GL additives. Drug release followed a linear pattern throughout dissolution after 1.5 days using PLGA and PLGA-HA. The amount of TR released after 1.5 days, irrespective of the type of formulation, was less than $8 \%$. Different formulations were achieved using the Taguchi method and we found that burst release decreased to $1.3 \%$ using TR (15\%), HA (1\%), and GL (3\%) formulations.

Analysis of in vitro and in vivo studies showed that TR concentration contributes most significantly to the control of burst release. The study of drug release continued over 125 days in vitro. This prolonged delivery period will enable decreased drug dosage and possibly reduce the TR's undesirable side effects. The PLGA-HA combination exhibited significant buffering capacity that prevented tissue inflammation at the injection site, which is the result of acidic degradation products of the PLGA membrane.

The structure of TR changed from crystalline to amorphous in the presence of GL, which increases TR's solubility in PLGA, resulting in low burst release. The drug release pattern observed during this study can be considered the result of developing a novel drug delivery system based on optimizing water-insoluble drug release, decreasing tissue inflammation and minimizing burst release. This system promises to be useful for routine drug delivery research particularly in regard to in situ gel formulation and burst control for high potency drugs.

\section{Acknowledgments}

The authors thank Exir Pharmaceutical Co. (Boroujerd, Iran) and Copenhagen University for financial support, and the latter for generously providing equipment.

\section{Disclosure}

The authors report no conflicts of interest in this work.

\section{References}

1. Kakinoki S, Taguchi T. Antitumor effect of an injectable in-situ forming drug delivery system composed of a novel tissue adhesive containing doxorubicin hydrochloride. Eur J Pharm Biopharm. 2007;67: 676-681.

2. Lee F, Chung JE, Kurisawa M. An injectable hyaluronic acid-tyramine hydrogel system for protein delivery. J Control Release. 2009;134: 186-193.

3. Fraylich MR, Liu R, Richardson SM, et al. Thermally-triggered gelation of PLGA dispersions: towards an injectable colloidal cell delivery system. J Colloid Interface Sci. 2010;344:61-69.

4. Hollister LE. Site-specific drug delivery to CNS: Old and new. Neurobiol Aging. 1989;10:628-631.

5. Levy RJ, Vinod L, Strickberger SA, Underwood T, Davis J. Controlled release implant dosage forms for cardiac arrhythmias: review and perspectives. Drug Delivery. 1996;3:137-142.

6. Tipton AJ, Dunn RL. In situ gelling systems. In: Senior J, Radomsky M, editors. Sustained Release Injectable Products. Englewood, CO: Interpharm Press; 2000:71-102.

7. Hatefi A, Amsden B. Biodegradable injectable in situ forming drug delivery systems. J Control Release. 2002;80:9-28.

8. Packhaeuser CB, Schnieders J, Oster CG, Kissel T. In situ forming parenteral drug delivery systems: an overview. Eur J Pharm Biopharm. 2004;58:445-455.

9. Dunn RL, English JP, Cowsar DR, Vanderbilt DP, inventors. Biodegradable in-situ forming implants and methods of producing the same. Patent 5733950. 1998 March 31.

10. Ravivarapu HB, Moyer KL, Dunn RL. Sustained suppression of pituitary-gonadal axis with an injectable, in situ forming implant of leuprolide acetate. J Pharml Sci. 2000;89:732-741.

11. Ravivarapu HB, Moyer KL, Dunn L. Parameters affecting the efficacy of a sustained release polymeric implant of leuprolide. Int J Pharm 2000;194:181-191.

12. Lambert WJ, Peck KD. Development of an in situ forming biodegradable poly-lactide-co-glycolide system for the controlled release of proteins. J Control Release. 1995;33:189-195.

13. Wang L, Kleiner L, Venkatraman S. Structure formation in injectable poly(lactide-co-glycolide) depots. J Control Release. 2003;90:345-354.

14. Kumari A, Kumar YS, Yadav SC. Biodegradable polymeric nanoparticles based drug delivery systems. Colloids Surf B Biointerfaces. 2010;75:1-18.

15. Luan X, Bodmeier R. Influence of the poly(lactide-co-glycolide) type on the leuprolide release from in situ forming microparticle systems. J Control Release. 2006;110:266-272.

16. Roy RK. A Primer on the Taguchi Method. New York: Van Nostrand Reinhold; 1990.

17. Fisher RA. The Design of Experiments. 8th ed. New York: Hafner Press; 1966.

18. Ashrafizadeh SN, Khorasani Z. Ammonia removal from aqueous solutions using hollow-fiber membrane contactors. Chem Eng J. 2010;162: 242-249.

19. Kuo HC, Jeng MC. Effects of part geometry and injection molding conditions on the tensile properties of ultra-high molecular weight polyethylene polymer. Mater Des. 2010;31:884-893.

20. Park SH. Robust design and Analysis for Quality Engineering. London, UK: Chapman and Hall; 1996.

21. Madaeni SS, Koocheki S. Application of taguchi method in the optimization of wastewater treatment using spiral-wound reverse osmosis element. Chem Eng J. 2006;119:37-44.

22. Joseph R, McGregor WJ, Martyn MT, Tanner KE, Coates PD. Effect of hydroxyapatite morphology/surface area on the rheology and processability of hydroxyapatite filled polyethylene composites. Biomaterials. 2002;23:4295-4302.

23. Birnbaum DT, Kosmala D, Henthorn B, Peppas B. Controlled release of beta-estradiol from PLAGA microparticles: the effect of organic phase solvent on encapsulation and release. J Control Release. 2000;65:375-387. 
24. Otsuka M, Udenodan H, Matsuda Y, et al. Therapeutic effect of in vivo sustained estradiol release from poly (lactide-co-glycolide) microspheres on bone mineral density of osteoporosis rats. Biomed Mater Eng. 2002;12:157-167.

25. Bese AV, Borulu N, Copur M, Colak S, Nuri Ata O. Optimization of dissolution of metals from Waelz sintering waste (WSW) by hydrochloric acid solutions. Chem Eng J. 2010;162:718-722.

26. Atkins PW. Physical Chemistry. 4th ed. Oxford, UK: Oxford University Press; 1990.

27. Kempe S, Metz H, Mader K. Do in situ forming PLG/NMP implants behave similar in vitro and in vivo? A non-invasive and quantitative EPR investigation on the mechanisms of the implant formation process. J Control Release. 2008;130:220-225.

28. Xu Q, Czernuszka JT. Controlled release of amoxicillin from hydroxyapatite-coated poly(lactic-co-glycolic acid) microspheres. J Control Release. 2008;127:146-153.

29. Lin PL, Fang HW, Tseng T, Lee WH. Effects of hydroxyapatite dosage on mechanical and biological behaviors of polylactic acid composite materials. Mater Lett. 2007;61:3009-3013.

30. Eliaz RE, Wallach D, Kost J. Delivery of soluble tumor necrosis factor receptor from in-situ forming PLGA implants: in-vivo. Pharm Res. 2000; 17:1546-1550.

31. Barakat NS. Optimization of physical characterization, skin permeation of naproxen from glycofurol-based topical gel. Asian J Pharm. 2010;4: 154-162.

32. Allhenn D, Lamprecht A. Microsphere preparation using the untoxic solvent glycofurol. Pharm Res. 2011;11:563-571.
33. Barakat N. Evaluation of glycofurol-based gel as a new vehicle for topical application of naproxen. AAPS Pharm Sci Tech. 2010;11:1138-1146.

34. Iwamoto R, Amiya S, Saito Y, Samura H. FT-NIR spectroscopic study of $\mathrm{OH}$ groups in ethylene-vinyl alcohol copolymer. Appl Spectrosc. 2001;55:864-870.

35. Suitchmezian V, Jess I, Nather C. Investigations on the polymorphism and pseudopolymorphism of the glucocorticoid triamcinolone: new findings for a well-known drug. Cryst Growth Des. 2007;7:69-74.

36. da Silva AA, de Matos JR, Formariz TP, et al. Thermal behavior and stability of biodegradable spray-dried microparticles containing triamcinolone. Intl J Pharm. 2009;368:45-55.

37. Young TH, Chuang WY, Wei CW, Tang CY. Investigation of the drug distribution and release characteristics from particulate membranes. J Memb Sci. 2001;191:199-205.

38. Stamatialis DF, Papenburg BJ, Girones M, et al. Medical applications of membranes: drug delivery, artificial organs and tissue engineering. J Memb Sci. 2008;308:1-34.

39. Ma D, McHugh AJ. The interplay of phase inversion and membrane formation in the drug release characteristics of a membrane-based delivery system. J Memb Sci. 2007;298:156-168.

40. Xiang A, Ma D, McHugh AJ. The interplay of phase inversion, polymer membrane formation, and drug release in a membrane-based delivery system. J Memb Sci. 2010;358:85-92.

41. McHugh AJ. The role of polymer membrane formation in sustained release drug delivery systems. J Control Release. 2005;109:211-221.
International Journal of Nanomedicine

\section{Publish your work in this journal}

The International Journal of Nanomedicine is an international, peerreviewed journal focusing on the application of nanotechnology in diagnostics, therapeutics, and drug delivery systems throughout the biomedical field. This journal is indexed on PubMed Central,

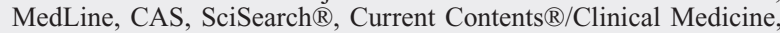

\section{Dovepress}

Journal Citation Reports/Science Edition, EMBase, Scopus and the Elsevier Bibliographic databases. The manuscript management system is completely online and includes a very quick and fair peer-review system, which is all easy to use. Visit http://www.dovepress.com/ testimonials.php to read real quotes from published authors 